\title{
Pacientes em rede: aspectos inovadores na plataforma PatientsLikeMe
}

E-Patients: innovative aspects in PatientsLikeMe platform

\section{Arquimedes Pessoni}

Professor do Programa de Mestrado em Comunicação e da graduação da Universidade Municipal de São Caetano do Sul. Pós-doutorando em Medicina na Faculdade de Medicina do ABC.

E-mail: pessoni@uscs.edu.br

Recebido em 7 de novembro de 2015. Aprovado em 10 de dezembro de 2015.

\section{Resumo}

O artigo busca apontar o novo papel desempenhado por pacientes na discussão de aspectos pessoais de saúde no ambiente digital. As inovações oferecidas pela plataforma PatientsLikeMe criam um ambiente onde pacientes, cuidadores e profissionais de saúde dividem dúvidas, sugestões e queixas que, trabalhadas de forma qualitativa, geram informações para pesquisadores do segmento médico e empresas farmacêuticas. A pesquisa mapeou a produção científica a partir da busca pela palavra-chave "Patientslikeme" e discute não apenas os aspectos inovadores da plataforma, mas também os interesses segmentados e desafios a vencer no novo locus comunicacional.

Palavras-chave: Comunicação e Saúde. Inovação. PatientsLikeMe.

\section{Abstract}

The article identifies the role of patients in discussing personal health issues in the digital environment. The innovations offered by the PatientsLikeMe platform create an environment where patients, caregivers and healthcare professionals share questions, suggestions and complaints that, studied qualitatively, can generate information for researchers from the medical field and pharmaceutical companies. The research maps the scientific production indexed using the keyword "PatientsLikeMe" and discusses not only the innovative aspects of the platform, but also the segmented interests and challenges to overcome in the new communicational locus.

Keywords: Health Communication. Innovation. PatientsLikeMe. 


\section{Introdução}

A tradicional relação entre pacientes e profissionais de saúde vem ganhando novos contornos a partir do advento da internet. A relação unidirecional em que o paciente desconhecia seus males e apenas o profissional da saúde decidia o que era melhor para cuidar/curar o doente deu lugar a um novo espaço de discussão, onde diferentes vozes podem ser ouvidas, desde o maior interessado pela cura - o paciente - ou seu cuidador que convive diariamente com o sofrimento, bem como os demais profissionais de saúde, que analisam o quadro do enfermo, fazem o diagnóstico e acompanham o tratamento.

Quando todos esses profissionais são reunidos em um só espaço coletivo, aquilo que é discutido - em forma de queixa, de diagnóstico, de medicamento utilizado, sucessos e fracassos de tratamento -, acaba por tornar-se um importante conteúdo para pesquisadores entenderem, em tempo real, como a doença pode ser combatida e como melhorar os efeitos colaterais dos medicamentos. Nesse sentido, de maneira inovadora, a plataforma PatientsLikeMe (PLM) reuniu esse público em um ambiente virtual, abriu as informações para pesquisadores e representantes de empresas farmacêuticas e o resultado tem sido bastante relevante para ambas as partes.

Frost et al. (2008) sugerem que a internet, por meio da plataforma PatientsLikeMe, serve tanto para facilitar a busca de informações sobre novos desenvolvimentos médicos como para conectar pacientes com diagnósticos parecidos. Os pacientes e cuidadores organizados em torno de dados médicos partilhados estão encontrando, de forma coletiva, informações para ajudar nas decisões de tratamento e conduzir a mudança na descoberta médica para melhorar o tratamento.

Para construirmos este artigo, partimos de uma das definições de inovação proposta por Rossetti (2013):

No campo da Comunicação, a inovação é mais evidente nas interfaces tecnológicas e nas novas mídias. Todavia, a inovação está presente também no impacto social das novas tecnologias de comunicação e informação, no novo receptor, agora produtor e interativo, nos processos cognitivos, nas linguagens, na estética e até mesmo em novas abordagens metodológicas e teóricas de comunicação. (ROSSETTI, 2013, p. 63).

Ainda tendo como referência a mesma autora, o que será mostrado na plataforma estudada pode representar também uma inovação parcial: "ocorre apenas em parte do objeto e, então, falamos em renovação, incremento, modificação, aperfeiçoamento, em que apenas um detalhe é novo" (ROSSETTI, 2013, p. 65). A metodologia utilizada foi 
a pesquisa bibliográfica tendo como palavra de busca "PatientsLikeMe" nos artigos da própria plataforma, no Portal de Periódicos Capes e Google Acadêmico, resultando 34 trabalhos internacionais no período de 2008 a 2015.

\section{Evolução na relação profissional de saúde-paciente}

Conforme explicam Sharma, Kilian e Leung (2014), o advento das redes sociais como uma importante plataforma para a interação humana introduziu uma nova dimensão para a relação médico-paciente, conhecido como Saúde 2.0. "O conceito de Saúde 2.0 é jovem e em evolução; até agora, tem significado o uso das mídias sociais pelos profissionais de saúde e pacientes para personalizar cuidados de saúde e promover a educação para a saúde" (SHARMA; KILIAN e LEUNG, 2014, p. 1).

Pessoni (2013) ressalta que a chegada da internet permitiu o surgimento de um novo paciente, mais participativo e empoderado, que chama para si parte da responsabilidade de escolher a forma como vai se relacionar com os profissionais de saúde e encontrar formas de tratamento:

O velho paradigma, no qual os profissionais de saúde eram vistos como os exclusivos detentores da sabedoria e do conhecimento, está gradualmente dando lugar a uma nova era da informação, em que a visão de mundo de pacientes, cuidadores e os sistemas de informação e relacionamento entre esses atores passam a ser vistos como muito importantes. Emerge o mundo do paciente-expert, também chamado e-patient (o neopaciente), ator que busca informações sobre saúde e doença com o auxílio da internet e, dessa forma, se mostra mais preparado para discutir com os profissionais de saúde sobre seu próprio tratamento. (PESSONI, 2013, p. 138).

A busca por mais informações na rede por parte dos pacientes ganha força quando esta se dá em grupos. Setz, Pereira e Naganuma (apud SANTOS et al., 2015, p. 64) salientam que fontes disponíveis como blogs, weblogs, páginas pessoais em redes sociais e grupos de apoio virtual produzem conhecimento baseado na experiência vivida de cada paciente, conhecimento esse que é trocado, compartilhado e multiplicado nas formas diversas de contato virtual.

Bletsos, Alexias e Tsekeris (2013) destacam que a internet é uma fonte valiosa de informação para os pacientes que procuram um melhor controle sobre o seu tratamento. Para os autores, isso significa que os doentes podem descobrir diferentes opções de tratamento e, como resultado, reduzir sua dependência na opinião de especialistas e ressaltam:

Ao ganhar mais controle sobre as questões relativas à sua saúde e sua relação com profissionais de saúde, os doentes têm agora a capacidade não só para obter informações relativas à sua 
doença, mas também para avaliar as habilidades de seus médicos, em comparação com as habilidades de outros médicos, como eles são apresentados por membros de comunidades virtuais (auto-organizadas). (BLETSOS; ALEXIAS e TSEKERIS, 2013, p. 138).

Tendo as comunidades virtuais de doentes como foco, Petersson e Erlinsdóttir (2015) enfatizam que o lançamento da Web. 2.0 elevou oportunidades para os pacientes construírem comunidades para compartilhar informações e criar a sua própria base de conhecimento, melhorando a capacitação dos doentes, alfabetização do paciente e, possivelmente, a participação do paciente e de cuidadores em decisões sobre sua saúde. Petersson e Erlinsdóttir acreditam que as comunidades virtuais auxiliam para que os pacientes possam ganhar mais informações sobre seu diagnóstico e suas doenças, bem como tirar conclusões sobre sua própria condição de saúde, com base em informações de outros pacientes, tomando decisões nem sempre informadas por seus médicos. Esse empoderamento dos pacientes digitais é ressaltado por Smith e Wicks (2008) quando afirmam que:

Como os consumidores ganharam maior acesso online para a literatura dos profissionais de saúde, eles também formaram suas próprias comunidades poderosas de conhecimentos, e assim a própria noção de "expertise” tem expansão sofrida. Tecnologias baseadas na internet têm grande potencial não só para capacitar os consumidores em geral, mas permitem que os pacientes possam fazer uma significativa contribuição para a discussão em curso de prestação de cuidados de saúde. (SMITH e WICKS, 2008, p. 682).

Reunir todos esses atores em uma só plataforma digital e dar acesso às informações por eles postadas para a indústria farmacêutica e pesquisadores de novos medicamentos foi uma atitude arriscada, mas que mostrou algumas vantagens. Assim nasceu a plataforma americana PatientsLikeMe.

\section{A plataforma PatientsLikeMe como espaço de pesquisa}

A plataforma PatientsLikeMe (PLM) foi cofundada em 2004 por três engenheiros do Massachusetts Institute of Technology (MIT): os irmãos Benjamin e James Heywood e um amigo de longa data, Jeff Cole, em Cambridge, Massachusetts. Cinco anos antes da criação, Stephen Heywood - irmão e amigo dos fundadores - foi diagnosticado com esclerose lateral amiotrófica (doença de Lou Gehrig), aos 29 anos. A família Heywood logo começou a procurar em todo o mundo iniciativas que poderiam prolongar e melhorar a vida de Stephen. Inspirado em experiências de Stephen, os cofundadores e equipe conceituada construíram uma plataforma de partilha de dados de saúde que poderia transformar 
a maneira como os pacientes pudessem administrar suas próprias condições de saúde e ajudar a indústria farmacêutica na forma de condução de pesquisas para melhorar a vida do paciente ${ }^{1}$. De acordo com informações disponíveis na plataforma, acessadas em novembro de 2015, dez anos após sua fundação, a PLM (Fig.1) reunia cerca de 350 mil membros, com mais de 2.500 enfermidades e condições de saúde registradas, 28 milhões de dados sobre doenças, que geraram 60 pesquisas científicas.

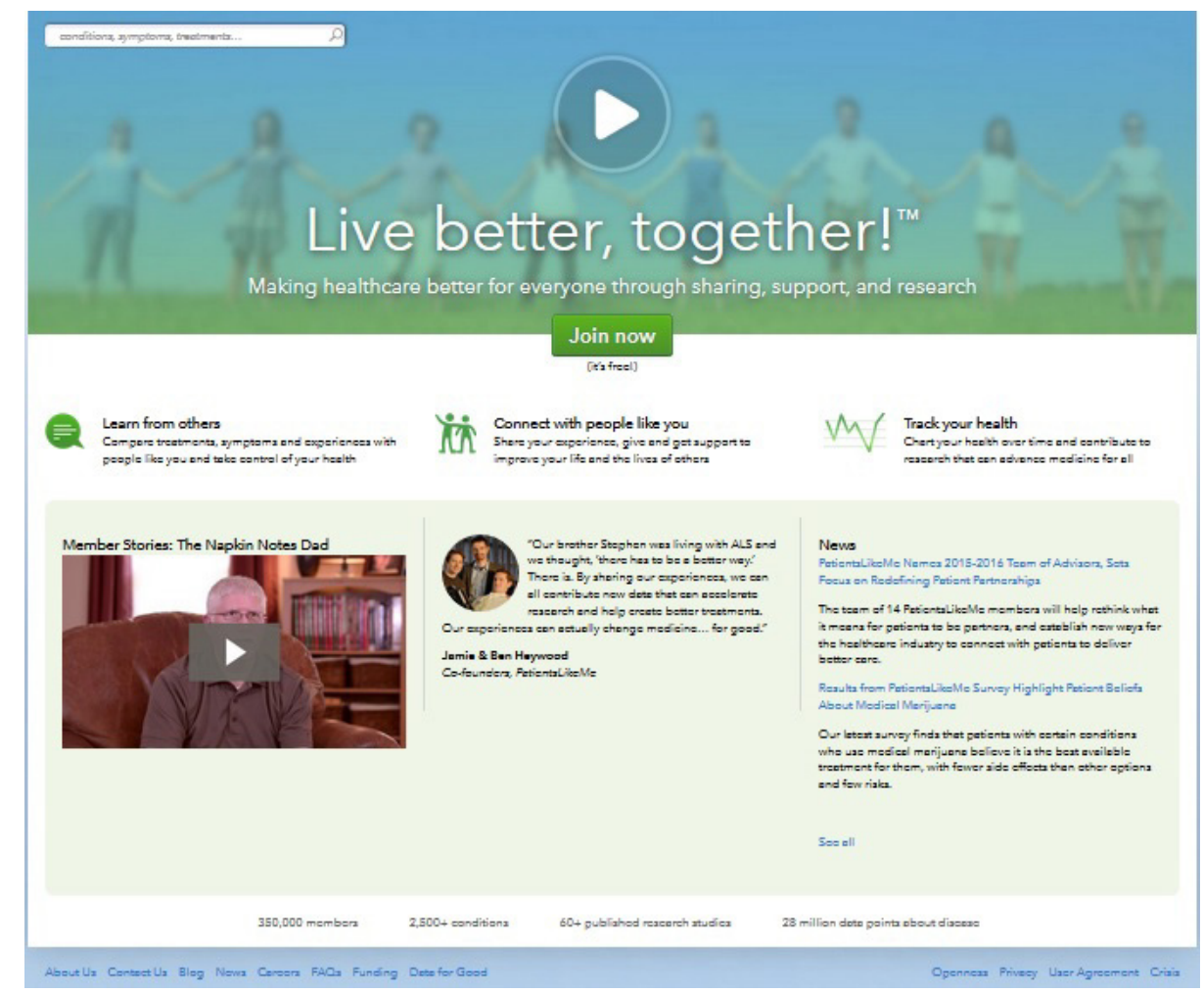

Figura 1. Página principal da plataforma PatientsLikeMe Fonte: www.patientslikeme.com. Acesso em: 04 nov. 2015

Frost e Massagli (2009) relatam que o lançamento da plataforma aconteceu em março de 2006. Acabou incorporando comunidades com pacientes portadores de outras doenças além de esclerose lateral amiotrófica: esclerose múltipla, doença de Parkinson, o HIV, fibromialgia, epilepsia e várias doenças neurológicas raras. Os autores explicam que os membros podem encontrar outras pessoas por variáveis demográficas, experiências de tratamento e histórias de sintomas e discutir os perfis e relatórios, bem como as preocupações gerais de saúde, por meio de fóruns, chats, mensagens privadas, além de postar comentários no perfil de outros pacientes (FROST e MASSAGLI, 2009, p. 227).

1 Informações disponíveis no link: <https://www.patientslikeme.com/about>. Acesso em 04 nov. 2015. 
Para Petersson e Erlinsdóttir (2015), a PLM foi projetada para uso dos pacientes independentes para obter informações e conhecimento sobre sua doença e de entrar em contato com outros pacientes com o mesmo diagnóstico para poder compartilhar experiências. O lucro da empresa vem de colaborações, incluindo empresas farmacêuticas, com a venda de dados do site para esses parceiros. De acordo com a plataforma, o objetivo geral dessas parcerias é aumentar as oportunidades para pesquisas. A empresa também tem colaborações com instituições acadêmicas e associações de pacientes. Os pacientes com determinados diagnósticos podem tornar-se membros de PLM sem custo algum. Citando a própria plataforma PatientsLikeMe, a adesão à PLM proporciona ao paciente oportunidade de entrar em contato com outros pacientes que têm diagnósticos semelhantes e experiências semelhantes ou com outro paciente com sua doença. As interações sociais tornam possível criar partilha de dados. Cada paciente no site é apresentado com dados demográficos. Há também descrições da maioria dos sintomas e tratamentos comuns para a doença. Os membros da comunidade são convidados a atualizar seu perfil semanalmente e postar mensagens nos fóruns. Os pacientes que optaram por um baixo grau de segurança durante seu cadastramento podem ser encontrados por não-membros das comunidades. Já os que optaram por um alto nível de confidencialidade e de segurança são apenas alcançáveis por outros membros da comunidade.

Tempini (2015) explica que o estilo da PLM por si só providencia o fornecimento de um combo-plataforma abrangente para a organização de sociabilidade e de defesa do paciente. Destina-se a tornar-se a rede de mídia social de escolha, na qual relações entre pacientes, profissionais clínicos, profissionais de saúde, empresas farmacêuticas, associações de doentes e organizações não-governamentais (ONGs ) são discutidos ou intermediados. Para Tempini, trata-se de um novo tipo de intermediário, representando a mais recente virada participativa na medicina.

Segundo Frost e Massagli (2008), os dados dos pacientes da PLM também são agregados individualmente para criar os recursos da comunidade, incluindo um relatório sobre a prevalência de cada tratamento e cada sintoma experimentado dentro do grupo. Membros discutem esses dados, bem como a saúde geral e preocupações por meio do fórum, mensagens privadas e comentários que eles postam nos perfis alheios. É justamente nesse caldo de informações geradas pelos pacientes que os pesquisadores podem obter conteúdo para realizar pesquisas de novos fármacos ou obter notificações sobre efeitos colaterais de medicamentos já consumidos. Também com grande quantidade de pacientes cadastrados com doenças específicas, a PLM representa um espaço privilegiado para captação de sujeitos de pesquisa para a indústria farmacêutica. 


\section{A pesquisa promovida pelos próprios pacientes}

Um exemplo de inovação no uso de informações disponibilizadas na plataforma PLM é relatada em artigo disponível na própria PLM e que também foi pauta da revista BusinessWeek. Frost et al. (2008) contam que em novembro de 2007, um paciente transmitiu uma notícia no fórum de debates sobre um estudo científico italiano pouco divulgado informando sobre um resultado promissor de lítio no tratamento da doença de Lou Gehrig. O paciente em questão, um analista de sistemas brasileiro, morador de Brasília (DF), teve acesso ao estudo antes da revisão científica formal pelos pares e processo de replicação em seres humanos. O ensaio italiano sugeriu que o lítio poderia ter um efeito benéfico para os pacientes com doença de Lou Gehrig. Ao socializar a informação num dos fóruns da PLM, conseguiu que mais de 400 pacientes com a doença se dispusessem a utilizar o lítio no tratamento da doença degenerativa. Empresas farmacêuticas acompanharam a ação e identificaram sucesso no composto, reativando a pesquisa italiana para fins de criação de novo medicamento.

A jornalista Catherine Arnst, autora da matéria da BusinessWeek, alertou sobre a nova forma de produção de pesquisa pelos próprios pacientes:

Para pacientes com expectativa de vida limitada, a capacidade de participar de uma forma rude de estudo, de baixo nível clínico sobre um novo tratamento é muito mais atraente e oportuna do que esperar por dados clínicos a serem publicados em literatura revisada por pares. Os pacientes podem checar diariamente com companheiros de dor e descobrir quais tratamentos funcionam, ou não, quais os sintomas que devem se preocupar, quais as ações a tomar. (ARNST, 2008, p. 2).

Epstein (2008, apud TEMPINI, 2015) ressalta que, para entender uma forma de organização inovadora como a representada pela PLM, é fundamental explicar as condições que moldam a produção de informação para além dos dados. No caso da PLM, Epstein explica que os pesquisadores não aprendem sobre os pacientes, suas experiências e suas situações de saúde em qualquer outra forma que não através dos dados sociais. Da forma como a plataforma PLM foi construída, as informações postadas diariamente pelos pacientes levam dados confiáveis para fora da vida do paciente e também para os pesquisadores. A quantidade de informação que pode ser expressa por dados recolhidos através de uma rede aberta permite traduzir o conhecimento a partir de contextos locais. Epstein evidencia o caráter coletivo e também comercial da plataforma:

O modelo de negócio da PatientsLikeMe está centrado em serviços de pesquisa comercial. Estes serviços são totalmente com base nos dados que o paciente (e outros membros) recolhe como parte 
de autoacompanhamento de atividades e interações com a comunidade de saúde, e giram em torno de tarefas de trabalhos complexos incluindo a agregação de dados, análise e elaboração de relatórios. Os clientes são organizações da indústria de cuidados de saúde, tais como empresas farmacêuticas ou planos de saúde. Através da venda de serviços, PatientsLikeMe assegura o financiamento para desenvolver o sistema informacional e para a pesquisa científica que a organização realiza e publica. A principal preocupação para a organização é reunir os melhores dados possíveis, ou seja, dados que informam, dizendo algo sobre uma experiência de vida ou evento que algum paciente está passando em algum lugar. (EPSTEIN, 2008, apud TEMPINI, 2015, p. 11).

O autor complementa, afirmando que, para os pacientes, o sistema representa uma forma possivelmente mais fácil de controlar sua saúde em detalhes, permitindo-lhes construir, ao longo do tempo, uma espécie de diário estruturado que armazena e resume vida no que tange à saúde. Mais importante ainda, os pacientes usam a rede para se conectar com outros pacientes como eles. Eles encontram apoio, oferecem ajuda, encontram regimes de tratamento alternativos na esperança de uma cura, informações sobre equipamentos e modificações de estilo de vida, pedir sugestões ou simplesmente comunicar seus sentimentos e experiências para alguém familiarizado com a sua dor. E exemplifica:

Isso pode significar muito para alguns pacientes, como aqueles que não se sentem compreendidos em seu contexto de vida (por exemplo, pacientes com fibromialgia), ou aqueles que não conhecem os peritos na sua doença, como os portadores de doenças raras, uma parcela relevante da população de doentes que talvez tenha recebido insuficiente atenção dos pesquisadores médicos. Para muitos pacientes, o site é um lugar para a partilha de dor e consolo. (EPSTEIN, 2008 apud TEMPINI, 2015, p. 11-12).

O autor comenta que a entrada de dados sobre o estado de saúde do paciente ao longo do tempo constrói uma história de saúde de sua vida em várias dimensões. Através de uma série de ferramentas de monitoramento, a PLM capta informações sobre os aspectos clínicos mais relevantes (por exemplo, sintomas, tratamentos, internações, qualidade de vida). As dimensões fundamentais da vida de saúde dos pacientes são capturadas através do rastreamento de condições (e eventos relacionados, por exemplo, diagnósticos, primeiros sintomas), de tratamentos (e afins parâmetros de dosagem, por exemplo, de drogas e de frequência), de sintomas (e gravidade relacionada), bem como as eventuais relações entre essas entidades (por exemplo, um sintoma associado a uma droga, como o seu efeito colateral). Há outras ferramentas para capturar aspectos outros de saúde, sejam genéricos (por exemplo, peso) ou 
específicos (testes de laboratório, por exemplo). Sem rastrear essas dimensões de saúde, pode-se dizer pouco sobre a experiência de vida dos pacientes. Epstein ressalta que o segredo do sucesso da plataforma é obter dados constantes dos pacientes, aquilo que realmente interessa aos pesquisadores:

A capacidade do sistema para recolher dados está dependente da sua capacidade para manter os pacientes envolvidos em tarefas de recolha de dados interativos. Ele [o sistema] precisa motivar os pacientes a voltar e continuar o autorelato. Pacientes envolvidos - visitando regularmente o site e participando de suas rotinas - permitem a recolha de dados longitudinais ao longo do tempo, tradicionalmente um conteúdo muito caro e característica valiosa para pesquisa. A necessidade de manter os pacientes envolvidos e introdução de dados ao longo do tempo caracterizam boa parte do esforço wpara desenvolver o sistema. É uma grande preocupação, uma vez que pacientes mal engajados podem omitir a entrada de informações clínicas importantes. (EPSTEIN, 2008, apud TEMPINI, 2015, p. 14).

Para incentivar os pacientes a inserir dados diários sobre suas condições de saúde, a plataforma PLM envia mensagens por e-mail e oferece "estrelas" na forma de pontuação para seus membros. Com mensagens intituladas "Today I feel" ("Hoje me sinto") os membros oferecem informações, matéria-prima essencial para o sucesso da plataforma, conforme podemos visualizar na figura 2 .

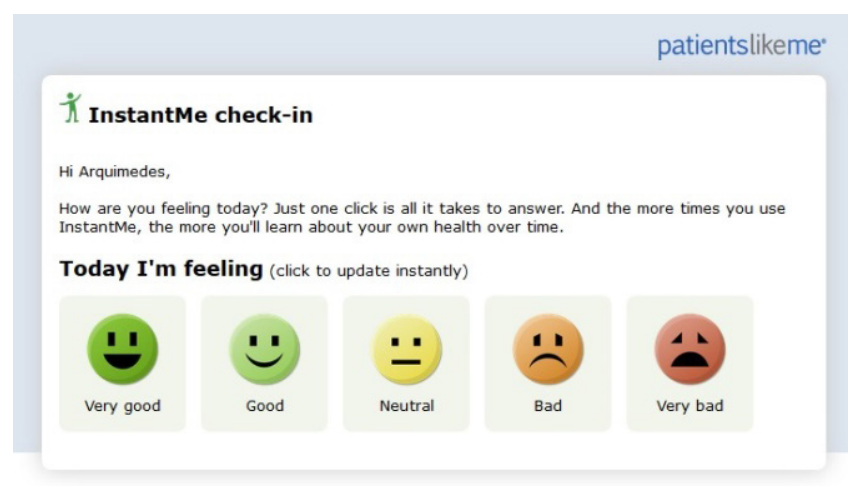

Figura 2. E-mail de convite para membros

Fonte: Acervo do autor

A Figura 3 mostra o modelo de prontuário eletrônico dos membros da PLM. À esquerda, acima, pode ser visualizada a seta que indica a quantidade de estrelas que o paciente possui no momento, que poderá ser ampliada de acordo com a frequência na plataforma e na quantidade de informações oferecidas. A obtenção da terceira estrela pode garantir brinde, como uma camiseta personalizada da PLM, forma de incentivo encontrada para captar mais informações. 


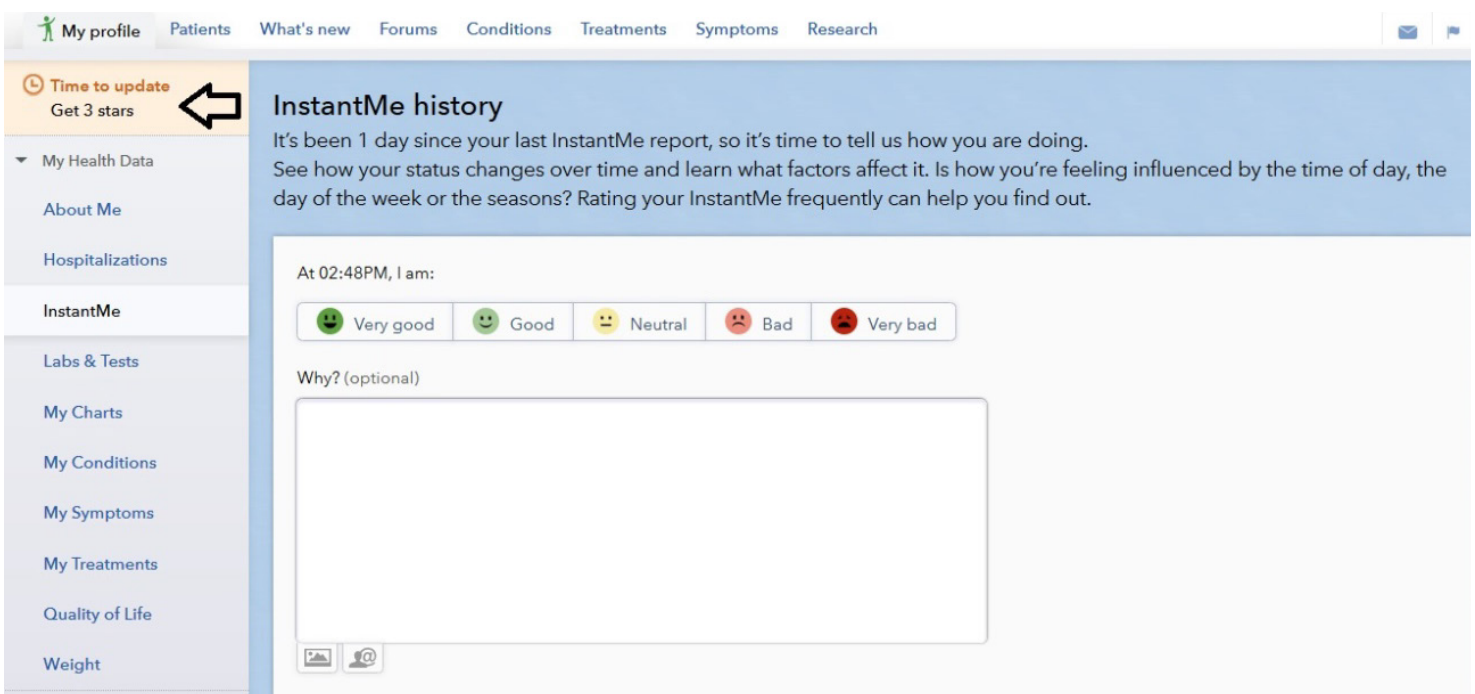

Figura 3. Prontuário do paciente

Fonte: Acervo do autor

\section{Desafios e novos cenários da comunicação e saúde online}

O futuro da relação de pacientes e profissionais de saúde aponta para os espaços digitais, na sociedade em rede, como preconiza Manuel Castells:

Todas as expressões culturais, da pior à melhor, da mais elitista a mais popular, vêm juntas nesse universo digital que liga, em um supertexto histórico gigantesco, as manifestações passadas, presentes e futuras da mente comunicativa. Com isso, elas constroem um novo ambiente simbólico. (CASTELLS, 1999, p. 394).

Nessa seara de informações pessoais sobre a saúde de pessoas fragilizadas por diversas enfermidades, a questão da privacidade precisa ser altamente levada em conta, conforme lembra Arnst (2008, p. 4): "E não importam quantas vezes os participantes dizem que eles não se importam quem sabe de suas doenças, há sempre o risco que os pacientes serão prejudicados quando a informação pessoal é exposta". Arnst cita uma fonte entrevistada para sua matéria: "A discriminação nos cuidados de saúde, habitação e emprego é um fato da vida. As consequências da divulgação podem ser muito graves", alerta Susan M. Dooha, diretora-executiva do Centro para a Independência das Pessoas com Deficiência, em Nova York (ARNST, 2008, p. 4).

Kallinikos e Tempini (2014) afirmam que a rede PLM coloca os doentes no centro da tarefa de geração de dados. Em assim fazendo, ela viola ou, de qualquer forma, arranha um dos costumes comuns dos médicos investigadores, em que a entrada de dados tem 
sido a prerrogativa exclusiva de especialistas (médicos e enfermeiros) como o resultado inevitável de pareceres de peritos. Em várias instâncias, a coleta de dados dos pacientes pode apresentar perda de qualidade. Van der Velden (2012) alerta que as pessoas tornam-se visíveis através dos dados que eles compartilham em redes digitais. Para a pesquisadora, informações pessoais, como as disponibilizadas na plataforma PLM, podem ser usadas como munição, por exemplo, para não obter um emprego futuro:

Um corpo visível é um corpo exposto, um corpo desprotegido que pode ser usado para atividades que não são consideradas sociais. Corpos de pacientes expostos podem ser quantificados, agregados e disponibilizados no mercado pelo operador para outras empresas. Várias comunidades online de pacientes são baseadas neste modelo. Corpos de pacientes expostos podem tornar-se organismos quantificáveis, que podem ser a medida de pressão, temperatura, peso, insulina, colesterol, uso de medicamentos, os efeitos secundários etc. (VAN DER VELDEN, 2012, p. 9).

De qualquer forma, os benefícios parecem pesar mais que os riscos e, para os pacientes, a busca pela cura ou pela melhoria da qualidade de vida pode justificar a exposição na rede. Encontrar novas formas de tratamento, fármacos recentes, apoio daqueles que vivem as mesmas condições limitadas e ainda ter a possibilidade de participar de pesquisas científicas que podem gerar uma substância que os ajudem na luta contra a doença justificam o risco. A comunicação em rede, de forma horizontal, mesmo que seja em ambiente virtual, é um ganho. O idioma ainda pode ser um empecilho para a captação de informações entre os que não se expressam em inglês, mas ferramentas de tradução online já estão minimizando esse problema.

Por outro lado, poder reunir em um só espaço pacientes do mundo inteiro que são portadores de doenças raras e que queiram oferecer informação atual, em tempo real sobre suas condições de saúde, representa uma oportunidade valiosa para pesquisadores da academia e das empresas de medicamentos.

A nova forma de relacionamento proposta pela PLM contribui para o empoderamento dos pacientes, uma vez que promove o surgimento do discurso do paciente contra o discurso médico, até então hegemônico e dominador. Contribui, também, para uma postura crítica contra médicos e consultórios de saúde. A mídia social, no caso da PLM, tem um papel importante na indústria de cuidados de saúde. O setor de saúde abriu um novo e eficaz canal de comunicação para os seus pacientes para compartilhar e trocar informações sobre a sua saúde pessoal e tratamento.

Enquanto fornece informação sobre sua saúde, o desenho comunicacional da plataforma PLM promove a troca de informações entre os pacientes. Esse suporte informativo 
e emocional representa duas dimensões principais do apoio social na área da saúde online. Informações - mesmo que de paciente para paciente - promovem mudanças de atitudes. Ter apoio de profissionais de saúde monitorando e dando apoio online por meio de chats, fóruns e mensagens pessoais pode representar soluções de dúvidas sem necessidade de deslocamentos até hospitais, minimizando custo e promovendo menos sofrimento a pacientes e cuidadores.

Num segundo momento, quando esse tipo de uso de comunicação online sair do âmbito particular para plataformas de governos, as informações coletadas poderão contribuir para a construção de políticas públicas voltadas a segmentos específicos, garantindo investimento maior em prevenção de doenças e promoção da saúde. As próprias campanhas de saúde serão beneficiadas, pois terão informações mais verdadeiras, direto das fontes, vindas dos pacientes para gestores. O custo dos investimentos em saúde poderá ser mais bem projetado a partir de demandas reais dos pacientes.

Weiner (2012) acredita que o processo de digitalização esteja bem encaminhado dentro de muitos sistemas de saúde. Para ele, ao longo das próximas décadas, em todos os rincões do globo, médicos, gestores, formuladores de políticas e cientistas irão trabalhar em estreita colaboração com os consumidores para planejar, projetar, desenvolver, implementar e avaliar a infraestrutura de $e$-saúde em constante expansão.

Huang, Jung e Liu (2014), tendo a realidade dos hospitais chineses como referência, acreditam que o crescimento de novas tecnologias de informação em saúde trarão uma revolução na comunicação moderna de assistência médica. "O desenvolvimento e implementação de ferramentas e-saúde podem aumentar o acesso dos usuários a informações sobre saúde e melhorar a qualidade de assistência médica" (HUANG, JUNG e LIU, 2014, p. 1). Para os pesquisadores, com uma população tão grande, com uma alta demanda por serviços de saúde e tantos hospitais e leitos hospitalares em uso, a execução de ferramentas e-saúde em sites hospitalares poderia melhorar muito a qualidade dos serviços de saúde, fornecendo auxilio para o paciente, capacitando os doentes em suas tomadas de decisão a respeito da saúde e reduzindo custos administrativos.

Para a PLM e empresas do ramo farmacêutico, a inovação na comunicação com os pacientes se mostra altamente vantajosa, conforme lembram Frost at al (2011). Ao longo dos últimos anos, os pacientes forneceram histórias de tratamento e avaliações sobre milhares de produtos médicos. Esses dados puderam auxiliar na avaliação da eficácia e segurança de alguns tratamentos de forma mais eficiente e ao longo de um período maior de tempo do que é naturalmente exequível através de ensaios tradicionais. Para os autores:

Os resultados relatados pelo paciente, como aqueles que entraram no PatientsLikeMe, oferecem uma abordagem única em tempo real para entender a utilização e o desempenho dos 
tratamentos através de muitas condições. Estes dados relatados pelo paciente podem fornecer uma nova fonte de evidências sobre usos secundários e potencialmente identificar alvos para tratamentos a serem estudados sistematicamente em ensaios de eficácia tradicionais. (FROST at al, 2011, p. 3).

Os pesquisadores acreditam que uma vantagem de coletar informações sobre o tratamento através de uma comunidade online representa a capacidade para chegar a uma grande população de usuários a um baixo custo. Na medida em que a internet torna-se mais acessível, uma população cada vez mais diversificada estará online e poderá participar de comunidades de apoio a pessoas com problemas de saúde.

\section{Considerações finais}

A inovação na comunicação proposta pela PatientsLikeMe aponta para a criação de um consultório virtual de dimensões mundiais, que poderá reunir experiências obtidas diretamente de pacientes, em quantidade e qualidade. Processar as informações e obter resultados que promovam a melhoria na qualidade de vida dos pacientes, adaptações de remédios que apresentam efeitos colaterais, geração de pesquisas de ponta e criação de vínculos entre pessoas com sofrimento físico ou mental serão várias das possibilidades desse novo modelo de negócio, impossível de ser pensado antes da internet e, a partir desse advento, viável e promissor.

A missão de transformar informação em comunicação e comunicação novamente em informação científica parece fechar o ciclo proposto pelo novo modelo de negócio promovido pela PLM. Se há o perigo da invasão de privacidade e possibilidade de vazamento de informações pessoais de saúde para outros fins que não a pesquisa, os riscos e ganhos devem ser avaliados e pesados pelos pacientes e seus cuidadores. As vantagens em dividir informações pessoais com tantas pessoas podem resultar em ganho de qualidade de vida, apoio social, empoderamento e, quiçá, cura. A decisão está na ponta dos dedos e a um clique, mas só pode ser tomada individualmente.

\section{Referências}

ARNST, C. Health 2.0: Patients as partners. BusinessWeek, dez. 2008. Disponível em: <http://ohm. ecce.admu.edu.ph/wiki/pub/Main/ResearchProjects/Health_2.0__Patients_as_Partners_-_ BusinessWeek.pdf>. Acesso em: 06 nov. 2015.

BLETSOS, K.; ALEXIAS, G.; TSEKERIS, C. Towards a fourth cosmology of doctor-patient relationship: a reflection on the virtual patient community PatientsLikeMe. TripleC, v. 11, n. 1, p. 136-144, 
2013. Disponível em: <http://www.triple-c.at/index.php/tripleC/article/view/462/445>. Acesso em: 06 nov. 2015.

CASTELLS, M. A sociedade em rede. São Paulo: Paz e Terra, 1999.

FROST, J.H.; MASSAGLI, M.P. Collaborative uses of personal health information: a study of PatientsLikeMe. In: CHI, 26., 2008, Florença, Itália.ACM 978-1-60558-012-8/08/04. Disponível em: $<$ http://citeseerx.ist.psu.edu/viewdoc/summary?doi=10.1.1.214.9272>. Acesso em: 06 nov. 2015.

FROST, J.H.; MASSAGLI, M.P.; WICKS, P; HEYWOOD, J. How the social web supports patient experimentation with a new therapy: the demand for patient-controlled and patient-centered informatics. In: AMIA ANNUAL SYMPOSIUM, 2008, Washington, DC. PatientsLikeMe, Cambridge, MA. Disponível em: <http://www.ncbi.nlm.nih.gov/pmc/articles/PMC2656086/ >. Acesso em: 06 nov. 2015.

FROST, J.; MASSAGLI, M. PatientsLikeMe the case for a data-centered patient community and how ALS patients use the community to inform treatment decisions and manage pulmonary health. Chronic Respiratory Disease, v. 6, n. 4, p. 225-229, 2009. Disponível em: <http://crd.sagepub.com/content/6/4/225.short >. Acesso em: 06 nov. 2015.

FROST, J.; OKUN, S.; VAUGHAN, T.; HEYWOOD, J.; WICKS, P. Patient-reported outcomes as a source of evidence in off-label prescribing: analysis of data from PatientsLikeMe. Journal of Medical Internet Research, v. 13, n. 1, 2011. Disponível em: <http://www.jmir.org/2011/1/e6/>. Acesso em: 6 nov. 2015.

HUANG, E.; JING, W.; LIU, T. Interactive e-health tools for patients on Chinese hospitals' websites. International Journal of Healthcare Management, v.. 7, n. 2, 2014. Disponível em: <http://www. maneyonline.com/doi/abs/10.1179/2047971914Y.0000000069>. Acesso em: 06 nov. 2015.

KALLINIKOS, J.; TEMPINI, N. Patient data as medical facts: social media practices as a foundation for medical knowledge creation. Information Systems Research, v. 25, n. 4, 2014. Disponível em: $<$ http://pubsonline.informs.org/doi/abs/10.1287/isre.2014.0544 >. Acesso em: 6 nov. 2015.

PESSONI, A. Inovações na comunicação entre neopacientes e profissionais da saúde. In: PESSONI, A.; PERAZZO, P. F. (Orgs.). Neorreceptor no fluxo da comunicação. Porto Alegre: EDIPUCRS, 2013. p. 137-152. (Coleção Comunicação \& Inovação, v.1). Disponível em: <http://ebooks.pucrs. br/edipucrs/Ebooks/Pdf/978-85-397-0403-3.pdf>. Acesso em: 06 nov. 2015.

PETERSSON, L.; ERLINSDÓTTIR, G. Will PatientsLikeMe.com affect the doctor- patient relation and the work environment of doctors? In: INTERNATIONAL ERGONOMICS ASSOCIATION CONGRESS (IEA), 19., 2015, Melbourne. Disponível em: <http://lup.lub.lu.se/luur/download?func $=$ downloadFile \&recordOId=8052835\&fileOId=8052837>. Acesso em: 6 nov. 2015 .

ROSSETTI, R. Categorias de inovação para os estudos em Comunicação. Comunicação \& Inovação, São Caetano do Sul, v. 14, n. 27, p. 63-72, 2013. 
SANTOS, G. S.; TAVARES, C. M. M.; FERREIRA, R. E.; COSME, S. F. P.. Rede social e virtual de apoio ao adolescente que convive com doença crônica: uma revisão integrativa. Aquichan, v. 15, n. 1, p. 60-74, 2015. Disponível em: <http://aquichan.unisabana.edu.co/index.php/aquichan/article/ view/3852>. Acesso em: 06 nov. 2015.

SHARMA, S.; KILIAN, R.; LEUNG, Fok-Han. Health 2.0 - Lessons learned: social networking with patients for health promotion. Journal of Primary Care \& Community Health, v. 5, n.3, p. 208-210, 2014. Disponível em: <http://jpc.sagepub.com/content/5/3/208.short>. Acesso em: 06 nov. 2015.

SMITH, C. A.; WICKS, P. J. PatientsLikeMe: consumer health vocabulary as a folksonomy. In: AMIA ANNUAL SYMPOSIUM, 2008, Washington, DC. PatientsLikeMe, Cambridge, MA. Disponível em: <http://www.ncbi.nlm.nih.gov/pmc/articles/PMC2656083/>. Acesso em: 06 nov. 2015.

TEMPINI, N. Governing PatientsLikeMe: information production and research through an open, distributed, and data-based social media network. The Information Society, v. 31, n. 2, p. 193-211, 2015. ISSN 0197-2243. Disponível em: <http://www.tandfonline.com/doi/full/10.1080/01972243.2015. 998108\#.VsTAsPIrLIU >. Acesso em: 6 nov. 2015.

VAN DER VELDEN, M. In/visible bodies: on patients and privacy in a networked world. In: CULTURAL ATTITUDES TOWARDS TECHNOLOGY AND COMMUNICATION, 8., 2012, Murdoch University, Australia. Disponível em: <https://www.duo.uio.no/handle/10852/41502>. Acesso em: 06 nov. 2015.

WEINER, J. P. Doctor-patient communication in the e-health era. Israel Journal of Health Policy Research, v. 1, n. 33, 2012. Disponível em: <http://www.biomedcentral.com/content/pdf/2045-4015-1-33. pdf>. Acesso em: 06 nov. 2015. 